PROCEEDINGS OF THE

AMERICAN MATHEMATICAL SOCIETY

Volume 140, Number 12, December 2012, Pages 4181-4186

S 0002-9939(2012)11266-3

Article electronically published on April 2, 2012

\title{
ON THE AUTOMORPHISMS OF THE SPECTRAL UNIT BALL
}

\author{
CONSTANTIN COSTARA
}

(Communicated by Thomas Schlumprecht)

Abstract. Let $\mathcal{A}$ be a (complex, unital) semisimple Banach algebra and denote by $\Omega_{\mathcal{A}}$ its open spectral unit ball, that is, the set

$$
\Omega_{\mathcal{A}}=\{a \in \mathcal{A}: \sigma(a) \subseteq \mathbf{D}\},
$$

where $\sigma(a)$ denotes the spectrum of $a$ in $\mathcal{A}$ and $\mathbf{D}$ is the open unit disc in the complex plane. We prove that if $F: \Omega_{\mathcal{A}} \rightarrow \Omega_{\mathcal{A}}$ is a holomorphic map satisfying $F(0)=0$ and $F^{\prime}(0)=I$ (the identity of $\mathcal{A}$ ), then for $a$ in $\Omega_{\mathcal{A}}$ the intersection of all closed discs lying inside $\mathbf{D}$ and containing $\sigma(a)$ equals the intersection of all closed discs lying inside $\mathbf{D}$ and containing $\sigma(F(a))$. When all the elements of $\mathcal{A}$ have an at most countable spectrum and $F$ is biholomorphic, this implies that $F$ preserves the convex hull of the spectrum. As an application of the same equality, we prove that if $\mathcal{B}$ is a semisimple Banach algebra and $T: \mathcal{A} \rightarrow \mathcal{B}$ is a unital surjective spectral isometry, then $\sigma(T(a))=\sigma(a)$ in the case when $\sigma(a)$ has exactly two elements.

\section{INTRODUCTION AND STATEMENT OF RESULTS}

Let $\mathcal{A}$ be a semisimple Banach algebra over the complex field $\mathbb{C}$ having unit $e$. Given $a \in \mathcal{A}$, we shall denote by $\sigma(a)$ its spectrum and by $\rho(a)$ its spectral radius. Also, by $\pi(a)$ we shall denote its peripheral spectrum, that is, the set of all $\lambda \in \mathbb{C}$ satisfying $|\lambda|=\rho(a)$. The open spectral unit ball of $\mathcal{A}$ is defined to be the set of all $a$ satisfying $\rho(a)<1$. The study of holomorphic maps on $\Omega_{\mathcal{A}}$ was motivated by the spectral Nevanlinna-Pick interpolation problem, which is of importance for control theory [3. Proving a Cartan type of theorem would be of great help in this case, and this leads us to consider holomorphic functions $F: \Omega_{\mathcal{A}} \rightarrow \Omega_{\mathcal{A}}$ with $F(0)=0$ and $F^{\prime}(0)=I$ (the identity on $\mathcal{A}$ ). If $F$ is such a map, then $F(c)=c$ for all $c$ in the centre of $\mathcal{A}$ [8, Theorem 1.a]. In particular, $F(\lambda e)=\lambda e$ for $|\lambda|<1$. Also, by [8. Theorem 3] we have that $F$ preserves the peripheral spectrum, that is,

$$
\pi(F(a))=\pi(a) \quad\left(a \in \Omega_{\mathcal{A}}\right) .
$$

Our first result is a slight generalization of (1.1). For $|\alpha|<1$ in $\mathbb{C}$, define $M_{\alpha}$ : $\Omega_{\mathcal{A}} \rightarrow \Omega_{\mathcal{A}}$ by putting

$$
M_{\alpha}(a)=(a+\alpha e)(e+\bar{\alpha} a)^{-1} \quad\left(a \in \Omega_{\mathcal{A}}\right) .
$$

Then the following result holds.

Received by the editors January 15, 2011 and, in revised form, May 18, 2011.

2010 Mathematics Subject Classification. Primary 46Hxx; Secondary 32Hxx, 47A10.

Key words and phrases. Spectrum, spectral unit ball, holomorphic mappings.

This work was supported by CNCSIS-UEFISCSU, project number 24/06.08.2010, PN II-RU Code $300 / 2010$.

(C)2012 American Mathematical Society Reverts to public domain 28 years from publication 
Theorem 1.1. Let $F: \Omega_{\mathcal{A}} \rightarrow \Omega_{\mathcal{A}}$ be holomorphic with $F(0)=0$ and $F^{\prime}(0)=I$. Given $|\alpha|<1$, we have

$$
\pi\left(F^{\prime}(\alpha e)(a)\right)=\pi(a) \quad(a \in \mathcal{A})
$$

and

$$
\pi\left(\left(M_{\alpha} \circ F\right)(a)\right)=\pi\left(M_{\alpha}(a)\right) \quad\left(a \in \Omega_{\mathcal{A}}\right) .
$$

What other spectral related sets, besides the peripheral spectrum, are preserved by such a map $F$ ? It was asked (see [8, Question 1]) whether it is spectrumpreserving on $\Omega_{\mathcal{A}}$. This is known to be true for example in the case when $\mathcal{A}$ is the set of all $n \times n$ complex matrices [9], or more generally, when $\mathcal{A}$ is finite dimensional [4, but the general case remains unsolved. Using Theorem 1.1 we obtain that for $a$ in $\Omega_{\mathcal{A}}$, the intersection of all closed discs lying inside $\mathbf{D}$ and containing $\sigma(a)$ equals the intersection of all closed discs lying inside $\mathbf{D}$ and containing $\sigma(F(a))$.

Corollary 1.2. Let $F: \Omega_{\mathcal{A}} \rightarrow \Omega_{\mathcal{A}}$ be holomorphic with $F(0)=0$ and $F^{\prime}(0)=I$. Suppose that $\bar{D}(z ; r)$ lies inside $\mathbf{D}$ and that $a \in \mathcal{A}$ satisfies $\sigma(a) \subseteq \bar{D}(z ; r)$. Then $\sigma(F(a)) \subseteq \bar{D}(z ; r)$. More than that, denoting by $\Gamma$ the circle of centre $z$ and radius $r$, then

$$
\sigma(a) \cap \Gamma=\sigma(F(a)) \cap \Gamma .
$$

In particular, for all a in $\Omega_{\mathcal{A}}$ we have

$$
\bigcap\{D \text { closed disc : } \sigma(F(a)) \subseteq D \subseteq \mathbf{D}\}=\bigcap\{D \text { closed disc : } \sigma(a) \subseteq D \subseteq \mathbf{D}\} .
$$

For $z \in \mathbb{C}$ and $r>0$, we have denoted by $D(z ; r)$ the open disk of centre $z$ and radius $r$ in $\mathbb{C}$, and by $\bar{D}(z ; r)$ the closed one.

Given an arbitrary $a \in \Omega_{\mathcal{A}}$, the intersection of all closed discs in $\mathbb{C}$ containing $\sigma(a)$ is the convex hull of $\sigma(a)$. It is quite natural then to ask whether we can eliminate the condition $D \subseteq \mathbf{D}$ in (1.4), since this would imply that $F$ preserves the convex hull of the spectrum. The next result shows that this can be done in the particular case of biholomorphic mappings acting on algebras having only elements with an at most countable spectrum.

Theorem 1.3. Let $F: \Omega_{\mathcal{A}} \rightarrow \Omega_{\mathcal{A}}$ be biholomorphic satisfying $F(0)=0$ and $F^{\prime}(0)=I$. Suppose also that each element of $\mathcal{A}$ has an at most countable spectrum. Then

$$
\operatorname{co}(\sigma(F(a)))=\operatorname{co}(\sigma(a)) \quad\left(a \in \Omega_{\mathcal{A}}\right),
$$

where co $(K)$ stands for the convex hull of $K \subseteq \mathbb{C}$.

An old problem of Kaplansky asks whether every surjective spectrum-preserving linear mapping between unital $C^{*}$-algebras has to be a Jordan morphism. Aupetit [2] showed that this is true in the case of von Neumann algebras. In fact, its proof shows that the same is true in the case when we are working with $C^{*}$-algebras of real rank zero [5. Theorem 1.1]. The main idea is to show that any such mapping sends projections into idempotents, and then to use the fact that in $C^{*}$-algebras of real rank zero any selfadjoint element is a limit of linear combinations of projections. The same problem can also be stated in the case of spectral isometries: is any spectral radius-preserving unital surjective linear mapping between unital $C^{*}$-algebras a Jordan morphism? This is still an open question even in the case of von Neumann algebras. By [6. Prop. 3.1] we know that in order to give a positive 
answer in the case of $C^{*}$-algebras of real rank zero, it is also sufficient to show that any such map sends projections into idempotents. Since unital surjective spectral isometries preserve the convex hull of the spectrum [7], the images of a non-trivial projection under such a map has as spectrum a subset of the interval $[0,1]$ on the real axis. A first step towards proving that the images of such projections are idempotents would be to show that their spectrum is exactly $\{0,1\}$. This comes from Theorem [1.4, which is the main result of this paper.

Theorem 1.4. Let $\mathcal{B}$ be a complex unital semisimple Banach algebra and $T: \mathcal{A} \rightarrow$ $\mathcal{B}$ a unital linear and onto mapping such that $\rho(T(a))=\rho(a)$ for every $a \in \mathcal{A}$. Then $\sigma(T(a))=\sigma(a)$ for every $a \in \mathcal{A}$ having at most two elements in its spectrum.

\section{Proofs}

Proof of Theorem 1.1. Given $|\alpha|<1$, the map $M_{\alpha}$ defined by (1.2) is an automorphism of the spectral unit ball, with $M_{\alpha}(0)=\alpha e, M_{\alpha}^{\prime}(0)=\left(1-|\alpha|^{2}\right) I$, $M_{\alpha}(-\alpha e)=0$ and $M_{\alpha}^{\prime}(-\alpha e)=\left(1-|\alpha|^{2}\right)^{-1} I$, its inverse being $M_{-\alpha}$. For $|\lambda|<1$, define $G_{\lambda}: \Omega_{\mathcal{A}} \rightarrow \Omega_{\mathcal{A}}$ by putting $G_{\lambda}=M_{-\lambda} \circ F \circ M_{\lambda}$. Since $F(\lambda e)=\lambda e$, then $G_{\lambda}(0)=0$. By the chain rule,

$$
\begin{aligned}
G_{\lambda}^{\prime}(0) & =M_{-\lambda}^{\prime}\left(F\left(M_{\lambda}(0)\right)\right) \circ F^{\prime}\left(M_{\lambda}(0)\right) \circ M_{\lambda}^{\prime}(0)=M_{-\lambda}^{\prime}(\lambda e) \circ F^{\prime}(\lambda e) \circ M_{\lambda}^{\prime}(0) \\
& =F^{\prime}(\lambda e) .
\end{aligned}
$$

The spectral Schwarz lemma [8, Theorem 3] gives $\rho\left(G_{\lambda}^{\prime}(0)(a)\right) \leq \rho(a)$, an inequality which holds for all $a$ in $\mathcal{A}$. That is, $\rho\left(F^{\prime}(\lambda e)(a)\right) \leq \rho(a)$ for all $a$ in $\mathcal{A}$. Now fix such an $a$, and consider the function $\lambda \mapsto \rho\left(F^{\prime}(\lambda e)(a)\right)$ on $\mathbf{D}$. It is subharmonic [1, Theorem 3.4.7], and by what we have just proved it is bounded by $\rho(a)$ on D. We also have $F^{\prime}(0)(a)=a$, and hence the value of the function at 0 is exactly $\rho(a)$. By the maximum principle for subharmonic functions [1, Theorem 3.4.11], we conclude that $\pi\left(F^{\prime}(\lambda e)(a)\right)=\pi(a)$ for all $\lambda \in \mathbf{D}$ and $a \in \mathcal{A}$. Thus $\pi\left(G_{\lambda}^{\prime}(0)(a)\right)=\pi(a)$ for all $a \in \mathcal{A}$, and then by the same spectral Schwarz lemma we conclude that $\pi\left(G_{\lambda}(a)\right)=\pi(a)$ for all $a \in \Omega_{\mathcal{A}}$. For $a \mapsto M_{-\lambda}(a)$, this gives $\pi\left(\left(M_{-\lambda} \circ F\right)(a)\right)=\pi\left(M_{-\lambda}(a)\right)$, an equality which holds for all $a$ in $\Omega_{\mathcal{A}}$ and for all complex $\lambda$ with $|\lambda|<1$.

If $\Gamma$ is a circle inside the open unit disc, then there exist $0<r<1$ and $\alpha \in \mathbf{D}$ such that the Möbius transformation $M_{\alpha}$ sends the circle $\Gamma_{r}$ of centre 0 and radius $r$ into $\Gamma$. Indeed, $M_{\alpha}$ sends $\Gamma_{r}$ into the circle of centre $\alpha\left(1-r^{2}\right) /\left(1-|\alpha|^{2} r^{2}\right)$ and radius $r\left(1-|\alpha|^{2}\right) /\left(1-|\alpha|^{2} r^{2}\right)$. Let $w \in \mathbf{D}$ be the centre of $\Gamma$ and consider the equation (in $\alpha$ )

$$
\alpha\left(1-r^{2}\right) /\left(1-|\alpha|^{2} r^{2}\right)=w .
$$

If $w=0$, then take $\alpha=0$. If $w \neq 0$, since $|\alpha|\left(1-r^{2}\right) /\left(1-|\alpha|^{2} r^{2}\right)=|w|$, then

$$
\alpha_{r}=\frac{-\left(1-r^{2}\right)+\sqrt{\left(1-r^{2}\right)^{2}+4 r^{2}|w|^{2}}}{2 r^{2}|w|} \cdot e^{i \arg (w)} \in \mathbf{D}
$$

is a solution for (2.1). When $r$ goes to 0 , the value of $r\left(1-\left|\alpha_{r}\right|^{2}\right) /\left(1-\left|\alpha_{r}\right|^{2} r^{2}\right)$ goes to 0 , and when $r$ goes to 1 the value of the same expression goes to $1-|w|$. By continuity, there exists $r$ between 0 and 1 such that the radius of $M_{\alpha_{r}}\left(\Gamma_{r}\right)$ is exactly the radius of $\Gamma$, and therefore $M_{\alpha_{r}}\left(\Gamma_{r}\right)=\Gamma$. 
Proof of Corollary 1.2. Let $\alpha \in \mathbf{D}$ be such that $M_{\alpha}$ sends $\Gamma$ into a circle of center 0 and radius $R \in(0,1)$. Let $a \in \mathcal{A}$ be such that $\sigma(a) \subseteq \bar{D}(z ; r)$. Then $\rho\left(M_{\alpha}(a)\right) \leq$ $R$. Now (1.3) gives $\rho\left(M_{\alpha}(F(a))\right) \leq R$, that is, $\sigma\left(M_{\alpha}(F(a))\right) \subseteq \bar{D}(0 ; R)$. Therefore $M_{\alpha}(\sigma(F(a))) \subseteq \bar{D}(0 ; R)$, which gives $\sigma(F(a)) \subseteq M_{\alpha}^{-1}(\bar{D}(0 ; R))=\bar{D}(z ; r)$. More than that, we have $\lambda \in \sigma(a) \cap \Gamma$ if and only if $M_{\alpha}(\lambda) \in \pi\left(M_{\alpha}(a)\right)$. That is, by (1.3) if and only if $M_{\alpha}(\lambda) \in \pi\left(M_{\alpha}(F(a))\right)$, which is equivalent to $\lambda \in \sigma(F(a)) \cap \Gamma$.

The proof of Theorem 1.3 is based on Corollary 1.2 and the following identity principle for countable analytic multifunctions: if $D$ is a domain in the complex plane $\mathbb{C}$ and $K$ an analytic multifunction on $D$ having the property that $K(\lambda) \subseteq \mathbb{C}$ is at most countable for each $\lambda \in D$, then for a fixed $z \in \mathbb{C}$ we have that $Z=$ $\{\lambda \in D: z \in K(\lambda)\}$ is either at most countable or it is all $D$ (see, for example, [1, Chapter VII]).

Proof of Theorem 1.3, Let $b \in \Omega_{\mathcal{A}}$ and consider $\lambda \in \mathbb{C}$ satisfying $|\lambda|+\rho(b)<1$. Therefore, the disc $\bar{D}(-\lambda ; \rho(b))$ lies inside $\mathbf{D}$. We have $b-\lambda e \in \Omega_{\mathcal{A}}$ and $\sigma(b-\lambda e) \subseteq$ $\bar{D}(-\lambda ; \rho(b))$. By Corollary 1.2 we have that $\sigma(F(b-\lambda e)) \subseteq \bar{D}(-\lambda ; \rho(b))$ and $\sigma(F(b-\lambda e)) \cap \Gamma=\sigma(b-\lambda e) \cap \Gamma$, where $\Gamma$ is the circle of centre $-\lambda$ and radius $\rho(b)$. This gives

$$
\pi(F(b-\lambda e)+\lambda e)=\pi(b) \quad\left(b \in \Omega_{\mathcal{A}} ; \rho(b)+|\lambda|<1\right) .
$$

Consider $a \in \Omega_{\mathcal{A}}$ and $\gamma \in \mathbb{C}$ satisfying $\rho(a)+2|\gamma|<1$; then by putting $\lambda=\gamma$ and $b=a+\gamma e$ we have $b \in \Omega_{\mathcal{A}}$ and $\rho(b)+|\lambda| \leq \rho(a)+2|\gamma|<1$. Then (2.2) gives

$$
\pi(F(a)+\gamma e)=\pi(a+\gamma e) \quad\left(a \in \Omega_{\mathcal{A}} ; \rho(a)+2|\gamma|<1\right) .
$$

For $0<|\mu| \leq 1$, the map $F_{\mu}(a):=F(\mu a) / \mu$ is well defined and analytic from $\Omega_{\mathcal{A}}$ into itself, having value 0 at 0 and derivative $I$ at 0 . Applying (2.3) to $F_{\mu}$ instead of $F$ we get

$$
\pi(F(\mu a) / \mu+\gamma e)=\pi(a+\gamma e) \quad\left(a \in \Omega_{\mathcal{A}} ; \rho(a)+2|\gamma|<1 ; 0<|\mu| \leq 1\right) .
$$

For $a \in \Omega_{\mathcal{A}}$ and $\gamma \in \mathbb{C}$ satisfying $\rho(a)+2|\gamma|<1$, consider the analytic multifunction $\mu \mapsto K(\mu):=\sigma(F(\mu a) / \mu+\gamma e)$, which is well defined on $\{\mu:|\mu|<1 / \rho(a)\}$ (its value at 0 is $\sigma(a+\gamma e))$. Pick an arbitrary $\alpha \in \pi(a+\gamma e)$. Then $\alpha \in K(\lambda)$ for $|\mu| \leq 1$. The identity principle gives then $\alpha \in K(\mu)$ for $|\mu|<1 / \rho(a)$, that is, $\alpha \in \sigma(F(\mu a) / \mu+\gamma e)$ for all $|\mu|<1 / \rho(a)$. Therefore, given any $a \in \Omega_{\mathcal{A}}$ we have

$$
\rho(F(\mu a) / \mu+\gamma e) \geq \rho(a+\gamma e) \quad(\rho(a)+2|\gamma|<1 ; \quad|\mu|<1 / \rho(a)) .
$$

This in turn implies that

$$
\rho(F(x)+z e) \geq \rho(x+z e) \quad\left(x \in \Omega_{\mathcal{A}} ; z \in \mathbb{C}\right) .
$$

Indeed, consider $x \in \Omega_{\mathcal{A}}$ and $z \in \mathbb{C}$ and choose $R>0$ such that $\rho(x) / R+2|z| / R<$ 1. Put $a=x / R, \mu=R$ and $\gamma=z / R$. Then $a \in \Omega_{\mathcal{A}}, \rho(a)+2|\gamma|=\rho(x) / R+$ $2|z| / R<1$ and $|\mu|=\rho(x) / \rho(a)<1 / \rho(a)$. Then (2.4) gives

$$
\begin{aligned}
\rho(x+z e) & =R \cdot \rho((x / R)+(z / R) e)=R \cdot \rho(a+\gamma e) \\
& \leq R \cdot \rho(F(R a) / R+\gamma e)=R \cdot \rho(F(R(x / R)) / R+(z / R) e) \\
& =\rho(F(x)+z e) .
\end{aligned}
$$

For all $a$ in $\mathcal{A}$, we have (see the proof of [1, Lemma 4.1.15])

$$
\operatorname{co}(\sigma(a))=\bigcap_{|z|>\rho(a)} \bar{D}(z, \rho(a-z e)) .
$$


Since $F$ preserves the spectral radius, by using (2.5) we obtain that for all $a \in \Omega_{\mathcal{A}}$,

$$
\begin{aligned}
\operatorname{co}(\sigma(a)) & =\bigcap_{|z|>\rho(a)} \bar{D}(z, \rho(a-z e)) \subseteq \bigcap_{|z|>\rho(F(a))} \bar{D}(z, \rho(F(a)-z e)) \\
& =\operatorname{co}(\sigma(F(a))) .
\end{aligned}
$$

Thus $\operatorname{co}(\sigma(a)) \subseteq \operatorname{co}(\sigma(F(a)))$ for all $a \in \Omega_{\mathcal{A}}$. Since $F$ is supposedly biholomorphic, the same also holds for its inverse $F^{-1}$. This gives $\operatorname{co}(\sigma(a)) \subseteq \operatorname{co}(\sigma(F(a)))$ for all $a \in \Omega_{\mathcal{A}}$.

What if we allow $\Gamma$ in the statement of Corollary 1.2 to touch the boundary of the unit disc? Let us say that $\Gamma$ is the circle with centre $z \in \mathbf{D}$ and radius $r \in(0,1)$ such that $|z|+r=1$, and suppose that $a \in \Omega_{\mathcal{A}}$ has its spectrum inside $\bar{D}(z ; r)$, with $\sigma(a) \cap \Gamma \neq \emptyset$. Then the intersection of all closed discs containing $\sigma(a)$ and lying inside $\mathbf{D}$ is a subset of $\bar{D}(z ; r)$, and using (1.4) we obtain that $\sigma(F(a)) \subseteq \bar{D}(z ; r)$ also. Considering $\left(r_{n}\right)_{n} \subseteq(0,1)$ such that $r_{n} \rightarrow 1$, then using Corollary 1.2 we obtain that $\sigma\left(F\left(r_{n} a\right)\right) \cap\left(r_{n} \Gamma\right)=\sigma\left(r_{n} a\right) \cap\left(r_{n} \Gamma\right)$. That is, $\sigma\left(F\left(r_{n} a\right) / r_{n}\right) \cap \Gamma=\sigma(a) \cap \Gamma$ for $n=1,2, \ldots$. So if $\alpha \in \sigma(a) \cap \Gamma$, then $F\left(r_{n} a\right) / r_{n}-$ $\alpha e$ is not invertible, for $n=1,2, \ldots$. Since $F\left(r_{n} a\right) / r_{n}-\alpha e \rightarrow F(a)-\alpha e$, using that the set of invertible elements in $\mathcal{A}$ is open we obtain that $F(a)-\alpha e$ is not invertible. That is, $\alpha \in \sigma(F(a))$. Thus $\sigma(a) \cap \Gamma \subseteq \sigma(F(a)) \cap \Gamma$. If the function $F$ is supposed to be biholomorphic, applying what we have just proved to $F^{-1}$ and $F(a)$, then $\sigma(F(a)) \cap \Gamma \subseteq \sigma\left(F^{-1}(F(a))\right) \cap \Gamma=\sigma(a) \cap \Gamma$. Thus, in this case we necessarily have $\sigma(F(a)) \subseteq \bar{D}(z ; r)$ and $\sigma(a) \cap \Gamma=\sigma(F(a)) \cap \Gamma$. We shall use this to prove our result on spectral isometries.

Proof of Theorem 1.4. From standard properties of unital, surjective spectral isometries, we have that $T$ is continuous and bijective, $T^{-1}: \mathcal{B} \rightarrow \mathcal{A}$ also being a spectral isometry. Also, $T$ preserves the peripheral spectrum and the convex hull of the spectrum 7 .

If the spectrum of $a \in \mathcal{A}$ has only one element, since $T$ preserves the convex hull of the spectrum we conclude that $T(a)$ has the same spectrum as $a$. Now suppose that $\sigma(a)$ has exactly two elements. Working with elements of the form $\alpha e+\beta a$ instead of $a$, with $\alpha \in \mathbb{C}$ and $\beta \in \mathbb{C} \backslash\{0\}$, we may suppose for example that $\sigma(a)=\{i, 1+i\}$. Let $H=\{z \in \mathbb{C}: \operatorname{Im}(z)>0\}$ and define $f: H \rightarrow \mathbf{D}$ by $f(z)=(z-i) /(z+i)$. Then $f$ is biholomorphic, its inverse being $g: \mathbf{D} \rightarrow H$ given by $g(w)=i(1+w) /(1-w)$. Define then $F: \Omega_{\mathcal{B}} \rightarrow \Omega_{\mathcal{B}}$ by the formula $F=T \circ f \circ T^{-1} \circ g$, that is,

$$
F(x)=T\left(\left[T^{-1}\left(i(e+x)(e-x)^{-1}\right)-i e\right]\left[T^{-1}\left(i(e+x)(e-x)^{-1}\right)+i e\right]^{-1}\right)
$$

for $x \in \Omega_{B}$. Let us observe that since $T$ and $T^{-1}$ preserve the convex hull of the spectrum, the spectral theorem for the holomorphic functional calculus implies that $F$ is well defined. Clearly, it is biholomorphic on $\Omega_{\mathcal{B}}$, with $F(0)=0$ and $F^{\prime}(0)=I$, the identity on $\mathcal{B}$.

Denote $b=T(a) \in \mathcal{B}$. Since $T$ preserves the peripheral spectrum, we have

$$
\pi(T(a-e / 2))=\pi(a-e / 2)=\{-1 / 2+i, 1 / 2+i\} .
$$

Thus $\{-1 / 2+i, 1 / 2+i\} \subseteq \sigma(b-e / 2)$, which gives $\{i, 1+i\} \subseteq \sigma(b)$. Therefore, $\{i, 1+i\} \subseteq \sigma(b) \subseteq[0,1]+i$, the last inclusion coming from the fact that $T$ preserves the convex hull of the spectrum. Thus $\sigma(b) \subseteq d$, where $d$ is the line $\mathbf{R}+i$ in the 
complex plane. Let us observe now that $f(d)$ is a circle $\Gamma$ in $\overline{\mathbf{D}}$ passing through 0 and 1. Using the remark following the proof of Corollary 1.2 we have that $\sigma(F(f(b)))$ lies inside $\Gamma$, with $\sigma(F(f(b))) \cap \Gamma=\sigma(f(b)) \cap \Gamma$. That is, $\sigma(T(f(a))) \cap \Gamma=$ $\sigma(f(b)) \cap \Gamma$. Let $z_{0}=1 / 2$ be the centre of $\Gamma$. Then

$$
\pi\left(T(f(a))-z_{0} e\right)=\pi\left(f(b)-z_{0} e\right) .
$$

Since $\sigma(f(a)) \cap \Gamma$ has exactly two elements, then $\pi\left(f(a)-z_{0} e\right)$ has exactly two elements. Since $T$ preserves the peripheral spectrum we have that $\pi\left(T\left(f(a)-z_{0} e\right)\right)=$ $\pi\left(f(a)-z_{0} e\right)$, and therefore $\pi\left(T(f(a))-z_{0} e\right)$ has exactly two elements. Thus $\pi\left(f(b)-z_{0} e\right)$ has exactly two elements, which means that $\sigma(f(b)) \cap \Gamma$ has exactly two elements. Thus $f(\sigma(b)) \cap \Gamma$ has two elements. This in turn implies that $\sigma(b)$ has exactly two elements on $d$, and therefore $\sigma(b)=\{i, 1+i\}$.

\section{REFERENCES}

1. B. Aupetit, A primer on spectral theory, Springer-Verlag, New York, 1991. MR 1083349 (92c:46001)

2. B. Aupetit, Spectrum-preserving linear mappings between Banach algebras or Jordan-Banach algebras, J. London Math. Soc. (2) 62 (2000), 917-924. MR1794294 (2001h:46078)

3. H. Bercovici, C. Foias and A. Tannenbaum, A spectral commutant lifting theorem, Trans. Amer. Math. Soc. 325 (1991), 741-763. MR1000144 (91j:47006)

4. C. Costara, A Cartan type theorem for finite-dimensional algebras, Linear Algebra Appl. 426 (2007), 299-304. MR2347842 (2008g:46068)

5. M. Mathieu, Spectrally bounded operators on simple $C^{*}$-algebras, II, Irish Math. Soc. Bull. 54 (2004), 33-40. MR.2138429 (2005m:47074)

6. M. Mathieu, Towards a non-selfadjoint version of Kadison's theorem, Annales Mathematicae et Informaticae 32 (2005), 87-94. MR2264870(2008a:46057)

7. M. Mathieu and A.R. Sourour, Hereditary properties of spectral isometries, Arch. Math. (Basel) 82 (2004), 222-229. MR2053626 (2004m:47073)

8. T.J. Ransford, A Cartan theorem for Banach algebras, Proc. Amer. Math. Soc. 124 (1996), No. 1, 243-247. MR1307559 (96d:46063)

9. T. Ransford and M. White, Spectral characterization of algebraic elements, Bull. London Math. Soc. 33 (2001), 77-82. MR.1798579 (2001j:46066)

Faculty of Mathematics and Informatics, Ovidius University, Mamaia Boulevard 124, 900527 Constanţa, Romania

E-mail address: cdcostara@univ-ovidius.ro 